\title{
Viral Hepatitis: Implication Of Viral Types from A To E
}

\author{
Chateen I Ali Pambuk*, Fatma Mustafa Muhammad and Mohammed Rhael Ali \\ University of Tikrit, Iraq \\ *Corresponding author: Chateen I Ali Pambuk, University of Tikrit, Iraq.
}

To Cite This Article: Chateen I Ali Pambuk. Viral Hepatitis: Implication Of Viral Types from A To E. Am J Biomed Sci \& Res. 2019 - 4(6). AJBSR. MS.ID.000852. DOI: 10.34297/AJBSR.2019.04.000852

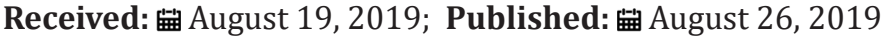

\begin{abstract}
Hepatitis is the emergence of inflammatory condition in the liver tissue as a result of viral infection. There are several different types of viral hepatitis as a result of the virus that causes the disease. In most cases, the source of the disease is the result of infection of three basic viruses' implication: the hepatitis A, B or C virus and with less frequent infections caused by Hepatitis D and E virus. Catching any of these viruses can lead to viral hepatitis, which accompanied by a wide different range of symptoms according to the virus implicated. Some of these infections are accompanied by mild and virtually inconceivable symptoms, but other symptoms may lead to liver breakdown, coma and death (if no alternative liver is found). The aim of this descriptive minireview, generally, is to shed light on the main types of virus involved in hepatitis

Keywords: Viral Hepatitis; Hepatitis A virus; Hepatitis Bvirus; Hepatitis C virus; Hepatitis D virus; Hepatitis E virus
\end{abstract}

\section{Introduction}

Viral Hepatitis is a contagious disease caused by a different type of viruses and causing damage to liver cells, it may be temporarily damage or permanent. Viral hepatitis is characterized by the presence of a profound inflammation in the liver tissue damaging hepatic cells [1]. Viral hepatitis infects the body causing jaundice (i.e. yellow skin), especially in children. There are five types of hepatitis are (A, B, C, D, E) There are also other types of non-classified or with non-obvious link with this disease, such as hepatitis virus $G$ ]1,2]. Some types of the infections are sexually transmitted, and the reason is common in others concerning the death of patients with hepatic viruses resulting in acute liver failure leading to coma and death. The inflammation in children less severe, but it may subsequently generate hepatic fibrosis and tissue damage to the liver or liver failure. There is no special treatment of viral hepatitis. When liver inflammation inflicted with hepatitis the viral infected cells die, which may lead to various complications, the patient may suffer recurrent bleeding due to lack of liver secretion of different coagulation factors (clotting factors). Hepatitis A is transmitted through contact with feces and urine and saliva of the infected patients. The hepatitis virus, hepatitis B C D infection transmitted via blood transfusions [1,3-5].

\section{Hepatitis A}

Hepatitis A is a highly contagious, but rarely fatal, the virus infects approximately 1.4 million people worldwide each year. It is a frequent infection among children in large and impoverished populations, and while traveling to countries where the prevalence rate of infection with hepatitis A virus is more than the incidence of typhoid fever [6,7]. The virus resides in the feces of people infected with hepatitis $\mathrm{A}$, and spreads the infection usually from person to person by eating and drinking contaminated with the virus spreads from an infected person, as the infection is transmitted by eating uncooked as some of the foods you eat raw, like oysters, vegetables, fruits that are eaten without peeling, or after washing food with contaminated water. Furthermore, it is caused by drinking contaminated snow or eating raw marine fish, vegetables and fruits (which did not wash well). Rarely, saliva, semen and vaginal secretions and urine cause the transmission of the disease $[7,8]$. The symptoms of hepatitis A shares similarity in the beginnings of the disease with the symptoms observed in influenza, infected children may not show any symptoms of the disease. The symptoms that appear in infected individual: feeling the pain of the body dark urine (tea colored), diarrhea, fever, weakness, nausea, vomiting and jaundice (yellowing of the skin and eye whites), loss of appetite, and light-colored stools [9].

Of the main symptoms of hepatitis A is severe drought as a result of vomiting and cluttered thinking and severe drowsiness or loss of consciousness and swelling of the face, hands, feet, ankles, legs and arms, and water retention in the body and bleeding nose or mouth and anal or under the skin [10]. Doctors advise hepatitis patients to eat high-calorie proteins while avoiding dehydration of the body, especially in the case of vomiting. Furthermore, advise them to take water and soup, fruit juice while avoiding alcohol and 
medications that may develops the liver damage. In the case of the emergence of itching skin it is advised to lubricate the involved area with soothing lubricants. Taking precautions is important when coming into contact with the patient or stools, wash hands with soap and hot water $[9,10]$. For prevention individuals can take the immunoglobulin vaccine (IG) within two weeks of the possibility of infection with hepatitis A virus. Hepatitis A vaccine has no effect in the case of a disease. The start of symptoms disappears within two weeks of the disease, which is contagious patient's stool. This virus type $\mathrm{A}$ is less dangerous than the rest [11].

\section{Hepatitis B}

Hepatitis B is considered a major global health problem. Hepatitis B is more infectious than HIV which causes AIDS virus. Hepatitis B is most commonly spread from mother to child at birth (perinatal transmission) or through horizontal transmission (exposure to infected blood), especially from an infected child to an uninfected child during the first 5 years of life. Moreover, Hepatitis B is also spread by percutaneous or mucosal exposure to infected blood and various body fluids, as well as through saliva, menstrual, vaginal, and seminal fluids. Sexual transmission of hepatitis B may occur, particularly in unvaccinated men who have sex with men and heterosexual persons with multiple sex partners $[12,13]$. Symptoms begin to appear after infection with 60-120 day. Symptoms appear only in $50 \%$ of infected adults. For infants and children, the percentage of the onset of symptoms are often less. Some people become very sick upon the onset of the infection. The disease symptoms can include jaundice (yellowing of the skin and eyes), dark urine turned to tea color, turning to the light-colored stool $[12,14]$.

The initial symptoms resemble influenza: loss of appetite, general weakness and fatigue, nausea and vomiting, fever, headache or pain in the joints and skin rash or itching, pain in the right upper abdomen food fat intolerances and cigarettes. The symptoms of hepatitis B is Similar to hepatitis A, in addition to the pain in the joints and muscles. In proportion ranging from $5-10 \%$ of the patients continue to have the virus as a chronic disease, and after several years the infection ended with liver fibrosis, the esophagus, stomach, become infected with varicose that cause vomiting and bloody, culminating in hepatic failure with incidence of liver tumors and cancer [13]. People if not infected with fulminant acute or chronic hepatitis B will recover from the disease within a week or a month. Children less vulnerable to the serious disease. More than $95 \%$ of adults and children who are exposed to the disease recover completely and without of any injury. The development of anti-bodies against the disease will be the hallmark to protect them from the disease in the future.

The disease can be transmitted to $5 \%$ of births by mother carriers of the virus. This class will recover from the disease. Among the $40 \%$ of people with one of the six people will be susceptible to liver cancer. Infection can be prevented by a vaccine for Hepatitis B [14-16].

\section{Hepatitis C}

Hepatitis C is mainly transmitted through infected blood or infected blood products. According to the World Health Organization, $80 \%$ of patients evolve to chronic hepatitis. And approximately 20 percent of them are involved with hepatic cirrhosis, then 5 percent of them develop liver cancer during the following ten years $[1,17]$. The infection appears in the form of acute hepatic involvement characterized by general malaise, loss of appetite, nausea, vomiting, physical pain and light fever, dark urine and Paul as well as skin irritation that was observed as a sign for each viral liver disease. The infection continues for several weeks after the patient begins to recover gradually in most cases, but there are some cases in which the damage occurs to the liver, hepatic failure and death may result [18].

Infection with hepatitis $\mathrm{C}$ in all parts of the world where more than 170 million people are infected with the virus. Most of them are infected with chronic liver disease. Chronic infection Boaradha or in conjunction with chronic liver disease may lead to cirrhosis of the liver after several years of illness. Also, this type of cases has dramatically led to liver cancer so ask the chronic hepatitis patients to avoid alcohol abuse as one of the accelerators in this dangerous disease occurs. Often it requires patients of chronic hepatitis $\mathrm{C}$ to liver transplantation [2,19]. The virus is transmitted exposure of subcutaneous blood contaminated and misuse of injections contaminated, particularly blood or acupuncture Chinese Ooalom or shaving or dental and dialysis equipment blades and the use of endoscopes transfer, as the virus from saliva is transmitted by a small percentage if encountered the presence of wounds Ochqqat inside the mouth. $20 \%$ of chronically infected people are infected with liver cirrhosis, including 1-4\% are infected liver cancer cells annually $[19,20]$.

\section{Hepatitis D}

The etiology also called delta virus (in English: Delta virus) cannot clone and reproduce only if there is another virus hepatitis $B$ virus, so the hepatitis $C$ virus (d) There appears always coupled with hepatitis C (b) [14] Hepatitis C is transmitted (d) through the blood transfer or its products or through sexual contact. Factors helping to move like the factors helping to spread the hepatitis B virus. And addicted to intravenous drugs are more injured. Infection can be prevented by preventing hepatitis B infection through vaccine viral hepatitis B [21,22].

\section{Hepatitis E}

It is one of the epidemic diseases linked to water pollution, and the virus is transmitted to humans through the mouth by contaminated food and drink. Because the virus comes out of the patient's body through the stool usually it is caused by infection 
of drinking water contaminated by sewage $[1,23]$. The incubation period of between two weeks and 9 weeks. The people between 15 40 years are more prone to infection. Pregnant women are more vulnerable, particularly to infection with the virus and are dying to have the percentage is much higher, perhaps up to $20 \%$ compared to less than $1 \%$ in others. There is no clinical difference between Hepatitis E and Hepatitis A. Causing virus (e) severe inflammation Qaibdia disappear automatically $[23,24]$.

\section{Conclusion}

Hepatitis A, B or C virus and with less frequent infections caused by Hepatitis D and E virus. Catching any of these viruses worldwide can lead to viral hepatitis, which accompanied by a wide different range of symptoms according to the virus implicated. Some of these infections are accompanied by mild and virtually inconceivable symptoms, but other symptoms may lead to liver breakdown, coma and death, the hepatitis accordingly is a risky worldwide disease with their different type's viruses.

\section{References}

1. Wasley A, Grytdal S, Gallagher K, Centers for Disease Control and Prevention (CDC) (2008) Surveillance for acute viral hepatitis United States, 2006. MMWR Surveill Summ 57(2): 1-24.

2. Previsani N, Lavanchy D (2002) World Health Organization: Hepatitis B (WHO/CDS/CSR/LYO/2002.2). p. 1-76.

3. (2017) Centers for Disease Control and Prevention. Viral Hepatitis Surveillance United States, 2014. p. 1-65.

4. (2017) Centers for Disease Control and Prevention. Surveillance for Viral Hepatitis-United States, 2015.

5. Pallavi K, Sravani D, Durga S, Durga S1, Pavan PNS, et al. (2017) Hepatitis: A Review on Current and Future Scenario. J In Silico In Vitro Pharmacol 3:1.

6. (2017) World Health Organization, Hepatitis A. pp. 328.

7. (2017) Centers for Disease Control and Prevention. Hepatitis A questions and answers for health professionals.
8. Blechová Z, Trojánek M, Kynčl J, Cástková J, John J et al. (2013) Clinical and laboratory features of viral hepatitis A in children. Wien Klin Wochenschr 125(3-4):83-90.

9. Jeong SH, Lee HS (2010) Hepatitis A: clinical manifestations and management. Intervirology 53(1): 15-19.

10. Samanta T, Das AK, Ganguly S (2010) Profile of hepatitis A infection with atypical manifestations in children. Indian J Gastroenterol 29(1):31-33.

11. Ott JJ, Irving G, Wiersma ST (2012) Long-term protective effects of hepatitis A vaccines. A systematic review. Vaccine 31(1): 3-11.

12. Ott JJ, Stevens GA, Groeger J, Wiersma ST (2012) Global epidemiology of hepatitis B virus infection: new estimates of age specific HBs Ag seroprevalence and endemicity. Vaccine 30(12): 2212-2219.

13. (2017) World Health Organization. Hepatitis B. 204.

14. Thomas E, Yoneda M, Schiff ER (2015) Viral hepatitis: past and future of HBV and HDV. Cold Spring Harb Perspect Med 5(2): a021345.

15. Nelson NP, Jamieson DJ, Murphy TV (2014) Prevention of perinatal hepatitis B virus transmission. J Pediatric Infect Dis Soc 3(suppl 1): S7-S12.

16. Cholongitas E, Tziomalos K, Pipili C (2015) Management of patients with hepatitis B in special populations. World J Gastroenterol 21(6): 17381748.

17. Chen SL, Morgan TR (2006) The natural history of hepatitis C virus (HCV) infection. Int J Med Sci 3(2): 47-52.

18. AASLD/IDSA HCV Guidance Panel (2015) Hepatitis C guidance: AASLD-IDSA recommendations for testing, managing, and treating adults infected with hepatitis C virus. Hepatology 62(3): 932-954.

19. (2017) World Health Organization. Hepatitis C. 164.

20. (2016) World Health Organization. Guidelines for the screening care and treatment of persons with chronic hepatitis $\mathrm{C}$ infection: updated version.

21. (2017) World Health Organization. Hepatitis D.

22. Sultanik P, Pol S (2016) Hepatitis delta virus: epidemiology, natural course and treatment. J Infect Dis Ther 34(271).

23. (2017) World Health Organization. Hepatitis E. 280.

24. Gerolami R, Moal V, Colson P (2008) Chronic hepatitis E with cirrhosis in a kidney-transplant recipient. N Engl J Med 358(8): 859-860. 